\title{
Determination of Excited-State Ionization Potentials for Lithium-Like Sequence Using Weakest Bound Electron Potential Model Theory
}

\author{
G. ÇELIKK, M. YILDIZ* AND H.Ş. KILIÇ \\ Department of Physics, Faculty of Arts and Science, Selcuk University, Campus \\ 42250 Konya, Turkey \\ (Received March 27, 2007; revised version July 30, 2007)

\begin{abstract}
In this work, the theoretical calculation of excited-state ionization potentials for $1 s^{2} 2 p^{2} P_{1 / 2}, 1 s^{2} 3 s^{2} S_{1 / 2}, 1 s^{2} 3 d^{2} D_{1 / 2}, 1 s^{2} 4 s^{2} S_{1 / 2}, 1 s^{2} 4 p^{2} P_{1 / 2}$, and $1 s^{2} 4 d^{2} D_{1 / 2}$ iso-spectrum series of lithium-like elements were carried out using a weakest bound electron potential model theory for nuclear charges from $Z=3$ to $Z=18$. The Breit-Pauli approximation was used for relativistic contributions. The obtained values are compared with the experimental results from literature. The overall agreement between data obtained in this work and experimental data from literature can appear to be quite good being generally within $0.1 \%$ of experimental values.
\end{abstract}

PACS numbers: 32.10.-f, 31.50.- $\mathrm{x}$

\section{Introduction}

Some considerable efforts have been made to determine the ionization potentials accurately for scientists studying physics, chemistry, and some other areas. They provide a crucial piece of information about the electronic structure and, therefore, are often used as benchmarks for theoretical methods [1]. The first ionization potential is a fundamental physical/chemical property of an element. An accurate determination of the first ionization potential connected directly to the atomic spectra is very important to know the element, interpret the systematic trends in the binding energies from element to element and put some conclusions about the electronic structure of atoms [2]. Since alkali atoms have only one outer electron energetically separated from the inner cores and have a simple electronic

*corresponding author; e-mail: muratyildiz@selcuk.edu.tr 
structure, they are popular subjects of theoretical and experimental investigations. This simplicity makes it possible to perform calculations in a very high accuracy tested by employing the comparison with known experimental level energies [3]. The accuracy of the data for lithium-like sequence is very important for understanding and interpretation of main physical and chemical processes. The calculation of the ionization potentials of atomic or ionic systems have been carried out using $R$-matrix method [4], multi-configuration Hartree-Fock (MCHF) method [5], relativistic configuration interaction (CI) method [6], relativistic many-body perturbation theory (MBPT) [7], and multi-configuration Dirac-Fock (MCDF) methods [8]. Recently, Zheng et al. have introduced a new method called the weakest bound electron potential model theory (WBEPMT) for the calculation of ionization potentials [9-12].

In this work, we employed a concept of iso-spectrum-level series in order to study the ionization potential of excited states in lithium-like sequence. While non-relativistic ionization potential of excited levels is derived from the WBEPM theory, the relativistic corrections of ionization potential in the Breit-Pauli approximation are expressed as a fourth-order polynomial in the nuclear charge $Z$.

\section{Theoretical method}

In many well-known theoretical methods, some configurations and orbital basis-set functions are required to obtain more accurate results for the high excited or ionized states and, therefore, this makes the calculation much more complicated. Studying the highly excited states or ionized states of atoms using the well-known $a b$ initio methods is also very difficult. The ionization potentials can, sometimes, be obtained using some experimental methods in atoms or ions but a theoretical calculation of the ionization potentials may not be performed so easily. Experimental determination of electronic energies with high accuracy is also not so easy. Therefore, in general, only a few first ionization potentials of a given iso-electronic series are available and can be reached easily [13]. However, theoretical and experimental works in the literature are concerned generally with low excited states and low ionized states rather than highly excited or ionized states.

In this paper, a semi-empirical method based on WBEPM theory and the concept of iso-spectrum-level series have been used for the accurate extrapolation of ionization potentials as a function of the nuclear charge. In this approximation, the ionization potentials of ground states can be studied along an iso-electronic series [9]. All atomic or ionic terms of the iso-electronic series have the same electron configuration. Thus, the concept of the iso-electronic series can only give some information associated with an electronic configuration and is unsuccessful to determine the ionization potentials of excited states. Therefore, the iso-spectrum-level series can conveniently be used in the determination of the ionization potential. The iso-spectrum-level series consists of some levels having the same level symbol in a given iso-electronic series. In this concept, the electron 
configuration, spectrum terms, and spectrum energy levels are all the same and the sole variable is the nuclear charge $Z$ [9].

The atomic and ionic energy can be given as a sum of non-relativistic energy and relativistic energy to be [9-12]:

$$
I(Z)=I_{\mathrm{nr}}(Z)+I_{\mathrm{r}}(Z),
$$

where $I_{\mathrm{nr}}(Z)$ and $I_{\mathrm{r}}(Z)$ are the non-relativistic and the relativistic potential, respectively, $I(Z)$ denotes the sum of non-relativistic and relativistic energies.

In this study, the non-relativistic potential $I_{\mathrm{nr}}(Z)$ was calculated using the WBEPM theory and the relativistic potential $I_{\mathrm{r}}(Z)$ was calculated using the BreitPauli approximation.

The WBEPM theory was developed by Zheng and has been applied to calculate various atomic properties, such as energy levels, ionization potentials, transition probabilities, oscillator strengths, and lifetime of excited levels in the many-electron atomic and ionic systems. This method is mainly based on the distinction of the weakest bound electron (WBE) and non-weakest bound electrons (NWBEs) in given atomic or ionic systems. By separation of the electrons in a given system, complex many-electron problems can be simplified as a single electron problem and then it can be solved more easily [14-19]. According to the WBEPM theory, the Schrödinger equations of the weakest bound electron under non-relativistic conditions are given as [16]:

$$
\left[-\frac{1}{2} \nabla^{2}+V\left(r_{i}\right)\right] \psi_{i}=\varepsilon_{i} \psi_{i},
$$

where $\psi_{i}$ is the wave vector for $i$-th electron, $\varepsilon_{i}$ is the energy eigenvalue of $i$-th electron, and $V\left(r_{i}\right)$ is

$$
V\left(r_{i}\right)=-\frac{Z^{*}}{r_{i}}+\frac{d(d+1)+2 d l}{2 r_{i}^{2}},
$$

where $Z^{*}$ is an effective nuclear charge, $n$ is the principal quantum number, $l$ is the angular momentum quantum number of the weakest bound electron. The effective principal quantum number $n^{*}$ and effective angular momentum quantum number $l^{*}$ are given in $d$ being defined to be $n^{*}=n+d$ and $l^{*}=l+d$. These are shortly defined below Eq. (3).

According to the WBEPM theory, the WBE moves in the potential field produced by the nucleus and the NWBEs. This potential field can be divided into two parts, one of which is the Coulomb potential and the other one is the dipole potential. Because of shielding effect of the NWBEs, the WBE is subjected to an effective nuclear charge $Z^{*}$. Since the WBE polarizes the atomic core, a dipole moment is produced. This dipole moment affects the behavior of the WBE and the potential function produced by this dipole moment can be defined as in Eq. (3). In this case, the principal quantum number $n$ and the angular momentum quantum number $l$ of the weakest bound electron are replaced by the effective principal quantum number $n^{*}$ and effective angular momentum quantum number $l^{*}$, respec- 
tively. The introduction of $d$ effectively modifies the integer quantum numbers $n$ and $l$ into the forms of non-integer momentum numbers to be $n^{*}=n+d$ and $l^{*}=l+d$. These terms differ from the usual core polarization potential which behaves asymptotically as $1 / r^{4}$, in which the effective dipole moment of the core is used as a parameter rather than being derived from the polarizability of the core in the electric field of the WBE [19].

In Eq. (3), $V\left(r_{i}\right)$ is the potential function produced by the non-weakest bound electrons and nucleus. Moreover, $Z^{*}$ is the effective nuclear charge, $r_{i}$ is the distance between $i$-th weakest bound electron and nucleus, and $l$ is the angular momentum number of the weakest bound electron. In this method, the effect of the screening of other electrons except for the weakest electron is not exact. Therefore, the Coulomb term of potential in the WBEPM theory is used as the effective nuclear charge $Z^{*}$ defined to be [9-12]:

$$
Z^{*}=\sqrt{(Z-\sigma)^{2}+g\left(Z-Z_{0}\right)} .
$$

The parameters given in Eq. (4) can be determined from the definition of the iso-spectrum-level series. Here $Z_{0}$ is the nuclear charge and $\sigma$ is the screening constant of the first term in the iso-spectrum-level series. $g$ is a relative increase factor that indicates the effect on the effective nuclear charge resulting from the increased nuclear charge in the series. The ionization potential of an atom or ion is given as [9-12]:

$$
I_{\mathrm{nr}}=\frac{Z^{*}}{2 n^{* 2}}=\frac{(Z-\sigma)^{2}+g\left(Z-Z_{0}\right)}{2 n^{* 2}} .
$$

As mentioned above, the excited state ionization potential is also not an invariant function of nuclear charge. The $n^{*}$ parameter can be determined by using the first difference of the non-relativistic ionization potential. If we plot the first differences of experimental ionization energies $\Delta I_{\exp }=I(Z+1)-I(Z)$ versus nuclear charge $Z$ in the iso-spectrum-level series, we find a linear relationship as shown in Fig. 1.

It can be clearly seen from Fig. 1 that the effective principal number $n^{*}$ is approximately constant in the iso-spectrum-level series and can be obtained simply from the slope of the curve. The parameter $\sigma$, the screening constant of the first term in the iso-spectrum-level series, is calculated from Eq. (5). The relative increase factor $g$ is the arithmetic average of the $g_{i}$ obtained from the same equation. We have employed each term given in the iso-spectrum-level series to obtain the parameters.

In this study, we have employed the Breit-Pauli approximation for the relativistic effects. The terms including relativistic effects increase approximately as the fourth power of nuclear charge $Z$ [20]. In order to improve accuracy and reliability on the ionization potential values, the relativistic corrections should be employed in the calculations. The relativistic corrections of the iso-spectrum-level series could be determined from a fourth-order polynomial in nuclear charge [10]: 


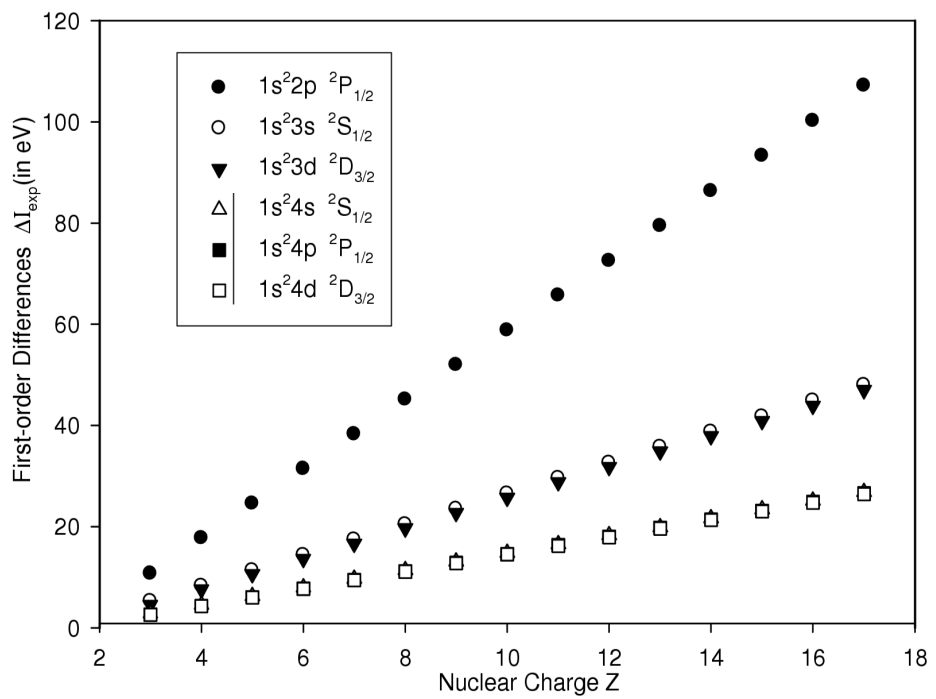

Fig. 1. A plot of the first differences of experimental ionization energies $\Delta I_{\exp }$ versus the nuclear charge $Z$ along some iso-spectrum level series of lithium-like sequence.

$$
I_{\mathrm{r}}=\sum_{i=0}^{4} a_{i} Z^{i}
$$

where $Z^{i}$ is used for the calculation of relativistic contribution and effective nuclear charge of relevant atom. According to Eq. (1), $I_{\mathrm{r}}=I-I_{\mathrm{nr}}$ and $a_{i}$ parameters can be easily obtained. In this study, $a_{i}$ parameters have been determined using the value of $I_{\exp }-I_{\mathrm{nr}}$. The value of $I_{\exp }$ is obtained from literature [21]. Thus, the ionization potential of the iso-spectrum-level series could be calculated from Eq. (7) [9-12]:

$$
I=\frac{(Z-\sigma)^{2}+g\left(Z-Z_{0}\right)}{2 n^{2}}+\sum_{i=0}^{4} a_{i} Z^{i}
$$

\section{Results and discussions}

We have studied the determination of the ionization potential of the excited states in the lithium-like sequence with $Z=3-18$ for $1 s^{2} 2 p^{2} P_{1 / 2}, 1 s^{2} 3 s^{2} S_{1 / 2}$, $1 s^{2} 3 d^{2} D_{3 / 2}, 1 s^{2} 4 s^{2} S_{1 / 2}, 1 s^{2} 4 p^{2} P_{1 / 2}, 1 s^{2} 4 d^{2} D_{3 / 2}$ series using the definition of iso-spectrum-level series, weakest bound electron potential model theory, and the Breit-Pauli approximation. The relativistic effects must be considered to obtain more accurate results for the ionization potential. The calculations of the ionization potentials of atomic or ionic systems have been performed using some powerful methods. In well-known theoretical methods, the ionization potentials of lithium-like sequences are studied for low excited states and low ionized states rather than for high excited or ionized states. The calculations are complex and difficult for the high excited and ionized states of lithium-like sequences. 
In general, the relativistic effects are less important for atoms having a low nuclear charge $Z$. For example, from $\mathrm{Li}$ atom to Ne atom, the relativistic corrections are about $0.01-0.1 \%$ of total energies [22, 23]. The main relativistic contributions to the ionization energy increase up to the fourth power of nuclear charge $Z$ of the iso-spectrum-level series [24]. Therefore, we employed Eq. (7) to correct the relativistic effects. In our calculation, the potential function in Eq. (3) represents the non-relativistic electrostatic potential acting on the WBE. Therefore, the energy eigenvalue of the WBE in Eq. (5), calculated using the potential function, should be of a negative value of the non-relativistic ionization energy of the WBE. However, the experimental data have been used to determine the relevant parameters given in Eq. (4).

TABLE I

Parameters needed for the calculation of the ionization potential of lithium-like sequence.

\begin{tabular}{c|c|c|c|c|c|c}
\hline \hline & $1 s^{2} 2 p^{2} P_{1 / 2}$ & $1 s^{2} 3 s^{2} S_{1 / 2}$ & $1 s^{2} 3 d^{2} D_{3 / 2}$ & $1 s^{2} 4 s^{2} S_{1 / 2}$ & $1 s^{2} 4 p^{2} P_{1 / 2}$ & $1 s^{2} 4 d^{2} D_{3 / 2}$ \\
\hline$\sigma$ & 1.984143 & 1.847497 & 1.999878 & 1.890647 & 1.990623 & 2.000057 \\
$n^{*}$ & 1.990163 & 2.991678 & 2.998572 & 3.991272 & 3.990919 & 3.997676 \\
$g$ & 0.108353 & 0.162396 & 0.001887 & 0.122831 & 0.055399 & 0.000603 \\
$a_{0}$ & 1.55965 & -0.225537 & 0.03933 & 0.1302626 & 0.09484 & -0.004741 \\
$a_{1}$ & -1.04008775 & 0.14802 & -0.032264 & -0.86905856 & -0.063476 & 0.0025421 \\
$a_{2}$ & 0.2264395 & -0.03128835 & 0.009515 & 0.0190275 & 0.013970 & -0.000320 \\
$a_{3}$ & -0.01928995 & 0.00252444 & -0.001214 & -0.00166 & -0.001229 & -0.00000516 \\
$a_{4}$ & 0.000536795 & -0.0000628983 & 0.0000568333 & 0.000050174 & 0.0000375170 & 0.0000015205
\end{tabular}

TABLE IIa

Non-relativistic ionization potential compared with experimental data $[\mathrm{eV}]$.

\begin{tabular}{c|c|c|c|c|c|c}
\hline \hline \multirow{2}{*}{$Z$} & \multicolumn{3}{|c|}{$1 s^{2} 2 p^{2} P_{1 / 2}$} & \multicolumn{3}{c}{$1 s^{2} 3 s^{2} S_{1 / 2}$} \\
\cline { 2 - 7 } & $I_{\exp }$ & $I_{\mathrm{nr}}$ & $\Delta I_{\exp -\mathrm{nr}}$ & $I_{\exp }$ & $I_{\mathrm{nr}}$ & $\Delta I_{\exp -\mathrm{nr}}$ \\
\hline 3 & 3.54344 & 3.54344 & 0 & 2.01833 & 2.01833 & 0 \\
4 & 14.25066 & 14.32546 & -0.07480 & 7.27085 & 7.28714 & -0.01629 \\
5 & 31.93084 & 31.97487 & -0.04403 & 15.58641 & 15.59502 & -0.00861 \\
6 & 56.49166 & 56.49166 & 0 & 26.94195 & 26.94197 & -0.00002 \\
7 & 87.90269 & 87.87583 & 0.02686 & 41.33234 & 41.32797 & 0.00437 \\
8 & 126.15448 & 126.12739 & 0.02709 & 58.75740 & 58.75303 & 0.00437 \\
9 & 171.24633 & 171.24633 & 0 & 79.21710 & 79.21715 & -0.00005 \\
10 & 223.18138 & 223.23266 & -0.05128 & 102.71914 & 102.72033 & -0.00119 \\
11 & 281.96689 & 282.08638 & -0.11949 & 129.25182 & 129.26256 & -0.01074 \\
12 & 347.61269 & 347.80748 & -0.19479 & 158.84802 & 158.84386 & 0.00416 \\
13 & 420.12125 & 420.39596 & -0.27471 & 191.47027 & 191.46422 & 0.00605 \\
14 & 499.54365 & 499.85183 & -0.30818 & 227.20910 & 227.12363 & 0.08547 \\
15 & 585.84889 & 586.17508 & -0.32612 & 265.95539 & 265.82211 & 0.13328 \\
16 & 679.10256 & 679.36572 & -0.26316 & 307.72651 & 307.55965 & 0.16686 \\
17 & 779.27514 & 779.42375 & -0.14861 & 352.60836 & 352.33624 & 0.27212 \\
18 & 886.40532 & 886.34916 & 0.05616 & 400.57306 & 400.15190 & 0.42116
\end{tabular}


TABLE IIb Non-relativistic ionization potential compared with experimental data $[\mathrm{eV}]$.

\begin{tabular}{c|c|c|c|c|c|c}
\hline \hline \multirow{2}{*}{$Z$} & \multicolumn{3}{|c|}{$1 s^{2} 3 d^{2} D_{1 / 2}$} & \multicolumn{3}{c}{$1 s^{2} 4 s^{2} S_{1 / 2}$} \\
\cline { 2 - 7 } & $I_{\exp }$ & $I_{\mathrm{nr}}$ & $\Delta I_{\exp -\mathrm{nr}}$ & $I_{\exp }$ & $I_{\mathrm{nr}}$ & $\Delta I_{\exp -\mathrm{nr}}$ \\
\hline 3 & 1.51291 & 1.51291 & 0 & 1.05064 & 1.05064 & 0 \\
4 & 6.05316 & 6.05379 & -0.00063 & 3.89488 & 3.90338 & -0.00850 \\
5 & 13.61943 & 13.61977 & -0.00034 & 8.45868 & 8.46357 & -0.00489 \\
6 & 24.21083 & 24.21085 & -0.00002 & 14.73119 & 14.73120 & -0.00001 \\
7 & 37.82680 & 37.82702 & -0.00022 & 22.70955 & 22.70627 & -0.00328 \\
8 & 54.46972 & 54.46831 & 0.00141 & 32.39465 & 32.38878 & 0.00587 \\
9 & 74.13465 & 74.13469 & -0.00004 & 43.78560 & 43.77874 & 0.00686 \\
10 & 96.81329 & 96.82617 & -0.01288 & 56.87938 & 56.87614 & 0.00324 \\
11 & 122.55134 & 122.54276 & 0.00858 & 71.68095 & 71.68099 & -0.00004 \\
12 & 151.31074 & 151.28445 & 0.02629 & 88.19848 & 88.19327 & 0.00521 \\
13 & 183.08382 & 183.05123 & 0.03259 & 106.52100 & 106.41300 & 0.10800 \\
14 & 217.92139 & 217.84312 & 0.07827 & 126.39435 & 126.34018 & 0.05417 \\
15 & 255.80362 & 255.66011 & 0.14351 & 148.01319 & 147.97479 & 0.03840 \\
16 & 296.67349 & 296.50221 & 0.17128 & 171.41096 & 171.31685 & 0.09411 \\
17 & 340.55864 & 340.36940 & 0.18924 & 196.51987 & 196.36636 & 0.15351 \\
18 & 387.53903 & 387.26170 & 0.27733 & 223.33434 & 223.12330 & 0.21104
\end{tabular}

TABLE IIC Non-relativistic ionization potential compared with experimental data $[\mathrm{eV}]$.

\begin{tabular}{c|c|c|c|c|c|c}
\hline \hline$Z$ & \multicolumn{3}{|c|}{$1 s^{2} 4 p^{2} P_{1 / 2}$} & \multicolumn{3}{|c}{$1 s^{2} 4 d^{2} D_{1 / 2}$} \\
\cline { 2 - 7 } & $I_{\exp }$ & $I_{\mathrm{nr}}$ & $\Delta I_{\exp -\mathrm{nr}}$ & $I_{\exp }$ & $I_{\mathrm{nr}}$ & $\Delta I_{\exp -\mathrm{nr}}$ \\
\hline 3 & 0.86995 & 0.86996 & -0.00001 & 0.85088 & 0.85089 & -0.00001 \\
4 & 3.48627 & 3.49489 & -0.00862 & 3.40437 & 3.40427 & 0.00010 \\
5 & 7.82257 & 7.82757 & -0.00500 & 7.65991 & 7.65963 & 0.00028 \\
6 & 13.86799 & 13.86800 & -0.00001 & 13.61696 & 13.61697 & -0.00001 \\
7 & 21.61960 & 21.61617 & 0.00343 & 21.27555 & 21.27628 & -0.00073 \\
8 & 31.07590 & 31.07209 & 0.00381 & 30.63627 & 30.63757 & -0.00130 \\
9 & 42.24083 & 42.23575 & 0.00508 & 41.70082 & 41.70084 & -0.00002 \\
10 & 55.10167 & 55.10716 & -0.00549 & 54.46076 & 54.46608 & -0.00532 \\
11 & 69.68630 & 69.68631 & -0.00001 & 68.96604 & 68.93331 & 0.03273 \\
12 & 86.02284 & 85.97321 & 0.04963 & 85.26550 & 85.10251 & 0.02404 \\
13 & 103.98956 & 103.96785 & 0.02171 & 102.99906 & 102.97368 & 0.02538 \\
14 & 123.74763 & 123.67024 & 0.07739 & 122.60092 & 122.54684 & 0.05408 \\
15 & 145.64399 & 145.08037 & 0.08402 & 143.86521 & 143.82197 & 0.04324 \\
16 & 168.27704 & 168.19825 & 0.07879 & 166.84025 & 166.79908 & 0.04117 \\
17 & 193.14794 & 193.02387 & 0.12407 & 191.56114 & 191.47816 & 0.08298 \\
18 & 219.73678 & 219.55724 & 0.17954 & 217.96899 & 217.85922 & 0.10977
\end{tabular}

In Table I the parameters of Eq. (4) for the relevant series are listed. Table II shows the present results and experimental energy values [21] for these series. The differences between the experimental energy values and our results are also given 
TABLE IIIa

Present results and comparison with experimental data $[\mathrm{eV}]$.

\begin{tabular}{c|c|c|c|c|c|c}
\hline \hline$Z$ & \multicolumn{3}{|c|}{$1 s^{2} 2 p^{2} P_{1 / 2}$} & \multicolumn{3}{c}{$1 s^{2} 3 s^{2} S_{1 / 2}$} \\
\cline { 2 - 7 } & $I_{\exp }$ & $I_{\text {present }}$ & $\Delta I$ & $I_{\text {exp }}$ & $I_{\text {present }}$ & $\Delta I$ \\
\hline 3 & 3.54344 & 3.54343 & 0.00001 & 2.01833 & 2.01832 & 0.00001 \\
4 & 14.25066 & 14.25065 & 0.00001 & 7.27085 & 7.29853 & -0.02768 \\
5 & 31.93084 & 31.91932 & 0.01152 & 15.58641 & 15.60361 & -0.01720 \\
6 & 56.49166 & 56.49166 & 0 & 26.94195 & 26.94193 & 0.00002 \\
7 & 87.90269 & 87.92279 & 0.02010 & 41.33234 & 41.32030 & 0.01204 \\
8 & 126.15448 & 126.18072 & 0.02624 & 58.75740 & 58.74408 & 0.01332 \\
9 & 171.24633 & 171.24632 & 0.00001 & 79.21710 & 79.21707 & 0.00003 \\
10 & 223.18138 & 223.11338 & 0.06800 & 102.71914 & 102.74161 & -0.02247 \\
11 & 281.96689 & 281.78853 & 0.17836 & 129.25182 & 129.31848 & -0.06666 \\
12 & 347.61269 & 347.29131 & 0.32156 & 158.84802 & 158.94701 & -0.09899 \\
13 & 420.12125 & 419.65412 & 0.46713 & 191.47027 & 191.62496 & -0.15469 \\
14 & 499.54365 & 498.92228 & 0.62137 & 227.20910 & 227.34861 & -0.13951 \\
15 & 585.84889 & 585.15396 & 0.69493 & 265.95539 & 266.11275 & -0.15736 \\
16 & 679.10256 & 678.42023 & 0.68233 & 307.72651 & 307.91061 & -0.18410 \\
17 & 779.27514 & 778.80505 & 0.47009 & 352.60836 & 352.73395 & -0.12559 \\
18 & 886.40532 & 886.40523 & 0.00009 & 400.57306 & 400.57301 & 0.00005
\end{tabular}

TABLE IIIb

Present results and comparison with experimental data $[\mathrm{eV}]$.

\begin{tabular}{c|c|c|c|c|c|c}
\hline \hline$Z$ & \multicolumn{3}{|c|}{$1 s^{2} 3 d^{2} D_{1 / 2}$} & \multicolumn{3}{|c}{$1 s^{2} 4 s^{2} S_{1 / 2}$} \\
\cline { 2 - 7 } & $I_{\text {exp }}$ & $I_{\text {present }}$ & $\Delta I$ & $I_{\exp }$ & $I_{\text {present }}$ & $\Delta I$ \\
\hline 3 & 1.51291 & 1.51290 & 0.00001 & 1.05064 & 1.05067 & -0.00003 \\
4 & 6.05316 & 6.05315 & 0.00001 & 3.89488 & 3.89706 & -0.00218 \\
5 & 13.61943 & 13.61942 & 0.00001 & 8.45868 & 8.45884 & -0.00016 \\
6 & 24.21083 & 24.21056 & 0.00027 & 14.73119 & 14.73148 & -0.00029 \\
7 & 37.82680 & 37.82679 & 0.00001 & 22.70955 & 22.71162 & -0.00207 \\
8 & 54.46972 & 54.46970 & 0.00002 & 32.39465 & 32.39714 & -0.00249 \\
9 & 74.13465 & 74.14223 & -0.00758 & 43.78560 & 43.78560 & -0.00153 \\
10 & 96.81329 & 96.84869 & -0.03540 & 56.87938 & 56.88183 & -0.00245 \\
11 & 122.55134 & 122.59476 & -0.04342 & 71.68095 & 71.68275 & -0.00180 \\
12 & 151.31074 & 151.38747 & -0.07673 & 88.19848 & 88.19255 & 0.00593 \\
13 & 183.08382 & 183.23522 & -0.15140 & 106.52100 & 106.41514 & 0.10586 \\
14 & 217.92139 & 218.14778 & -0.22639 & 126.39435 & 126.35560 & 0.03875 \\
15 & 255.80362 & 256.13629 & -0.33267 & 148.01319 & 148.02022 & -0.00703 \\
16 & 296.67349 & 297.21323 & -0.53974 & 171.41096 & 171.41652 & -0.00556 \\
17 & 340.55864 & 341.39246 & -0.83382 & 196.51987 & 196.55319 & -0.03332 \\
18 & 387.53903 & 388.51915 & -0.98012 & 223.33434 & 223.44014 & -0.10580
\end{tabular}

in Table II. Table III shows the present result $I_{\text {present }}$ including the relativistic corrections and these are displayed in comparison with compiled experimental values in column 2 for each sequence. In this study, the deviations $\Delta I_{\exp \text {-present }}$ between our results and experimental data are $\leq 0.09 \mathrm{eV}$ for nuclear charge $Z=$ 
TABLE IIIc

Present results and comparison with experimental data $[\mathrm{eV}]$.

\begin{tabular}{c|c|c|c|c|c|c}
\hline \hline$Z$ & \multicolumn{3}{|c|}{$1 s^{2} 4 p^{2} P_{1 / 2}$} & \multicolumn{3}{|c}{$1 s^{2} 4 d^{2} D_{1 / 2}$} \\
\cline { 2 - 7 } & $I_{\exp }$ & $I_{\text {present }}$ & $\Delta I$ & $I_{\exp }$ & $I_{\text {present }}$ & $\Delta I$ \\
\hline 3 & 0.86995 & 0.86995 & 0 & 0.85088 & 0.85087 & 0.00001 \\
4 & 3.48627 & 3.49029 & -0.00402 & 3.40437 & 3.40463 & -0.00026 \\
5 & 7.82257 & 7.82410 & -0.00153 & 7.65991 & 7.65990 & 0.00001 \\
6 & 13.86799 & 13.86806 & -0.00007 & 13.61696 & 13.61681 & 0.00015 \\
7 & 21.61960 & 21.61973 & -0.00013 & 21.27555 & 21.27553 & 0.00002 \\
8 & 31.07590 & 31.07762 & -0.00172 & 30.63627 & 30.63627 & 0 \\
9 & 42.24083 & 42.24108 & -0.00025 & 41.70082 & 41.69927 & 0.00155 \\
10 & 55.10167 & 55.11041 & -0.00874 & 54.46076 & 54.46480 & -0.00404 \\
11 & 69.68630 & 69.68677 & -0.00047 & 68.96604 & 68.93320 & 0.03284 \\
12 & 86.02284 & 85.97225 & 0.05059 & 85.12655 & 85.10480 & 0.02175 \\
13 & 103.98956 & 103.96984 & 0.01972 & 102.99906 & 102.97999 & 0.01907 \\
14 & 123.74763 & 123.68341 & 0.06422 & 122.60092 & 122.55922 & 0.04170 \\
15 & 145.16439 & 145.11774 & 0.04665 & 143.86521 & 143.84292 & 0.02229 \\
16 & 168.27704 & 168.27852 & -0.00148 & 166.84025 & 166.83160 & 0.00865 \\
17 & 193.14794 & 193.17232 & -0.02438 & 191.56114 & 191.52579 & 0.03535 \\
18 & 219.73678 & 219.80664 & -0.06986 & 217.96899 & 217.92607 & 0.04292
\end{tabular}

$3-18$ and $\leq 0.184 \mathrm{eV}$ for $Z=13-17$ in the $1 s^{2} 3 s^{2} S_{1 / 2}$ series; $\leq 0.07 \mathrm{eV}$ for $Z=3-12$ and $\leq 0.98 \mathrm{eV}$ for $Z=13-17$ in the $1 s^{2} 3 d^{2} D_{1 / 2}$ series; $\leq 0.038 \mathrm{eV}$ for $Z=3-18$ and $\leq 0.105 \mathrm{eV}$ for $Z=3-18$ in $1 s^{2} 4 s^{2} S_{1 / 2}$ series; $\leq 0.06 \mathrm{eV}$ for $Z=3-18$ in the $1 s^{2} 4 p^{2} P_{1 / 2}$ and $\leq 0.042 \mathrm{eV}$ for $Z=3-18$ in $1 s^{2} 4 d^{2} D_{1 / 2}$ series.

It can be seen that the present results are in good agreement with experimental energy values for these series. In all of these series, the experimental data from $Z=3$ to $Z=18$ are used to obtain the parameters and our results from $Z=3$ to $Z=18$ are calculated from Eq. (6). The results calculated in this work present a good agreement with experimental data and the agreement is generally within $0.1 \%$. According to the results obtained in this work, the approximation that the excited state ionization potentials are non-invariant functions of nuclear charge $Z$ is sufficient and successful for ionization potential calculations. The results are simply and accurately obtained using this method.

\section{Acknowledgments}

The authors gratefully acknowledge the Selçuk University Scientific Research Projects (BAP) Coordinating Office for financial support, project No. 06401024.

\section{References}

[1] H.P. Loock, L.M. Beaty, B. Simard, Phys. Rev. A 59, 873 (1999).

[2] K. Fuke, S. Yoshida, Eur. Phys. J. D 9, 123 (1999).

[3] E. Eliav, M.J. Vilkas, Y. Ishikawa, U. Kaldor, Chem. Phys. 311, 163 (2005). 
[4] J.A. Fernley, A. Hibbert, A.E. Kingston, M.J. Seaton, J. Phys. B, At. Mol. Opt. Phys. 32, 5507 (1999).

[5] G. Tachiev, C.F. Fischer, J. Phys. B, At. Mol. Opt. Phys. 33, 2419 (2000).

[6] K.M. Aggarwal, A. Hibbert, F.P. Kenan, Astrophys. J. Suppl. 108, 393 (1997).

[7] U.I. Safronova, W.R. Johnson, A.E. Livingston, Phys. Rev. A 60, 996 (1999).

[8] M.J. Vilkas, Y. Ishikawa, K. Koc, Int. J. Quant. Chem. 70, 813 (1998).

[9] N.W. Zheng, T. Zhou, T. Wang, R.Y. Yang, Y.J. Sun, F. Wang, C.G. Chen, Phys. Rev. A 65, 052510 (2002).

[10] N.W. Zheng, T. Wang, Chem. Phys. Lett. 376, 557 (2003).

[11] N.W. Zheng, T. Wang, Int. J. Quant. Chem. 93, 344 (2003).

[12] N.W. Zheng, T. Wang, Int. J. Quant. Chem. 98, 495 (2004).

[13] B. Elden, J. Chem. Phys. 33, 98 (1960).

[14] N.W. Zheng, A New Outline of Atomic Theory, Jiang Su Education Press, Nanjing 1988.

[15] N.W. Zheng, Chin. Sci. Bull. 22, 531 (1977); 33, 916 (1988).

[16] N.W. Zheng, T. Wang, D.-X. Ma, T. Zhou, J. Fan, Int. J. Quant. Chem. 98, 281 (2004).

[17] N.W. Zheng, T. Wang, Chem. Phys. 282, 31 (2002).

[18] N.W. Zheng, T. Wang, R.Y. Yang, J. Chem. Phys. 113, 6169 (2000).

[19] N.W. Zheng, T. Wang, T. Zhou, D.X. Ma, J. Phys. Soc. Jpn. 71, 1672 (2002).

[20] M.S. Safronova, U.I. Safronova, R. Brush, Phys. Lett. A 194, 106 (1994).

[21] J.R. Fuhr, W.C. Martin, A. Musgrove, J. Sugar, W.L. Wiese, NIST "Atomic Spectra Database", Version 2.0, World Wide Web URL: http://physics.nist.gov. Select "Physical Reference Data", 2006.

[22] A. Veillard, E. Clementi, J. Chem. Phys. 49, 2415 (1968).

[23] J.B. Mann, W.R. Johnson, Phys. Rev. A 4, 41 (1971).

[24] R.D. Cowan, The Theory of Atomic Structure and Spectra, University of California Press, Berkeley 1981. 